\title{
Sliding Mode Control for Electric Power Assist Systems"
}

\author{
Makoto YOKOYAMA $^{* *}$, Takafumi KAWASAKI ${ }^{* * *}$ and Masahiko TSUCHIYA ${ }^{* *}$ \\ **Department of Mechinical \& Production Engineering, Niigata University, \\ 8050 Ikarashi 2-nocho, Niigata, 950-2181, Japan \\ E-mail:m.yoko@eng.niigata-u.ac.jp \\ ${ }^{* * *}$ Canon Inc., \\ 30-30-2 Shimomaruko, Ohta-ku, Tokyo, 146-0092, Japan \\ E-mail: takafumi.kawasaki@canon.co.jp
}

\begin{abstract}
This paper presents a nonlinear controller for electric power assist systems based on the sliding mode control. The proposed sliding mode controller is designed to achieve desired nonlinear properties including gravitational effect with robustness against disturbances such as friction force and modeling errors. Furthermore, so-called reaching phase is positively utilized so that the operator can feel disturbance torque of relatively large amplitude which should be noticed as information about environment, for example, when hitting an obstacle accidentally. Although the reaching phase can be designed from several points of view, the dynamics in the reaching phase is linearlized in order to use fruitful linear control theories such as H-infinity theory.
\end{abstract}

Key words: Power Assist, Sliding Mode Control, Reaching Phase, Nonlinear Control, Robust Control, Servo Mechanism

\section{Introduction}

Power assist systems have been developed in a wide range of fields for many years such as space engineering, manufacturing, or nursing ${ }^{(1)-(6)}$. Although several kinds of actuators are used for these systems, an electric motor is one of common actuators. For example, electric power steering systems for automobiles have recently taken the place of the conventional hydraulic systems. In controller design for some of these systems, there are particular difficulties unlike fully automated systems. One of them is a man-machine interface problem which has been investigated rigorously by many researchers ${ }^{(7)}$. Another problem is that friction force in reduction-gears or cogging torque deteriorates the maneuverability or operator's feeling. This paper discusses the latter problem.

The theory of sliding mode control has applied to various control systems, since there are many excellent properties in the sliding mode, such as robustness against large variations in the system parameters and disturbances. However, these properties are only obtained during sliding motion. In the other hand, the system can also behave in another phase referred to as the reaching phase. In general, the controller is designed to make the time of reaching phase as short as possible. On the contrary this paper proposes a new control strategy of utilizing this reaching phase positively in order for the operator to get some important information about the environment or accident such as hitting an obstacle. For example, it is not easy for a driver of an automobile with a conventional power steering equipment to notice the fact that a tire hits a connection stone by accident. The proposed controller makes it possible for the operator to notice the accident by feeling the reaction 
force in such an emergency.

The rest of this paper is organized as follows. In Section 2, plant model and the desired closed-loop properties are described. In order to achieve these properties, a sliding mode controller is designed in Section 3. Since the system behavior against disturbances is very important in this study, the integral sliding mode control $^{(8),(9)}$ (ISMC), which can be seen a kind of disturbance observer, is employed. Some results from numerical simulations are presented in Section 4. Section 5 concludes the paper.

\section{Plant model and desired closed-loop properties}

In order to discuss the basic problems concerning electric power assist systems, the pant to be controlled is assumed to be a simple manipulator with a single degree of freedom as shown in Fig.1.

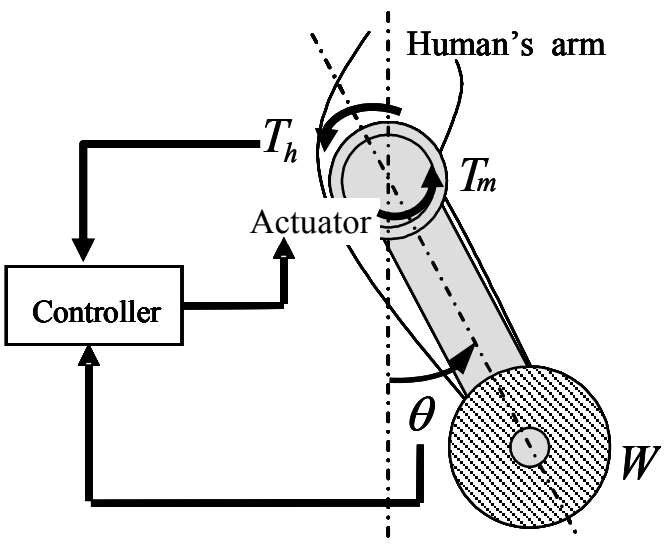

Fig.1 Schematic of power assist system

The actuator, which consists of a DC-motor and a set of reduction gears, assists an operator in lifting something heavy. The motion equation of this plant is given by

$$
J_{e} \ddot{\theta}=-W \sin \theta+T_{h}+n T_{m}+T_{d}
$$

where

$\theta:$ arm angle, $T_{h}$ : operation torque, $T_{m}:$ motor torque, $T_{d}$ : disturbance torque,

$J_{e}$ : equivalent inertia including all rotating parts, $n$ : gear ratio,

$W$ : equivalent load torque including equipment,

Eliminating the coil inductance in the motor, the motor torque is given by

$$
T_{m}=-\frac{n K_{t} K_{v}}{R} \dot{\theta}+\frac{K_{t}}{R} u
$$

where $K_{t}, K_{v}, R$ and $u$ denote the torque constant, the back emf constant, the resistance and the control voltage respectively. Substituting Eq.(2) into Eq.(1), the mathematical model of the system is written as

$$
J_{e} \ddot{\theta}=-n^{2} \frac{K_{t} K_{v}}{R} \dot{\theta}-W \sin \theta+T_{h}+\frac{n K_{t}}{R} u+T_{d}
$$

For this assist system, various desired properties in the closed loop can be considered from some points of view such as maneuverability, operator's feeling, stability, or actuator saturation. As a primary property, it can be considered that the operator lifting a load of its mass $m$ can feel as if he lifted just the load of its mass $\alpha m(0<\alpha<1)$ without the equipment. In this case, the ideal closed loop dynamics is given by

$$
m l^{2} \ddot{\theta}=-m g l \sin \theta+\frac{1}{\alpha} T_{h}
$$

where $l$ denotes the arm length, and $1 / \alpha$ will be referred to as a power amplification factor. From our previous study, however, the dynamics given by Eq.(4) is very sensitive to 
modeling errors and estimation errors of the plant state since its equilibrium point is a center point. Furthermore, the nonlinear term due to the gravity prevents to use a design method in frequency domain. First, we thus consider the transfer function of the linearlized model around the equilibrium point as follows:

$$
\frac{\Theta(s)}{T_{h}(s)}=\frac{1 / \alpha}{m l^{2} s^{2}+m g l}
$$

Adding a damping factor to provide robust stability, it follows that

$$
\frac{\Theta(s)}{T_{h}(s)}=\frac{G}{s^{2}+2 \zeta \omega_{0} s+\omega_{0}^{2}}
$$

The parameter $G$ is determined such that the DC gain should agree with that of Eq.(5), resulting in

$$
G=\frac{\omega_{0}^{2}}{\alpha m g l}
$$

The natural frequency $\omega_{0}$ and the damping coefficient $\zeta$ is determined such that Eq.(6) should approximate the gain property of Eq.(5), taking into account of the stability and the bandwidth corresponding to given tasks. A typical example will be described in Section 4 . Finally, changing $\theta$ to $\sin \theta$ so as to provide natural feeling due to the gravity, the desired closed-loop dynamics can be written by

$$
\ddot{\theta}=-\omega_{0}^{2} \sin \theta-2 \zeta \omega_{0} \dot{\theta}+G T_{h}
$$

which is asymptotically stable for $|\theta|<\pi$.

Using the state vector, $x=[\theta, \dot{\theta}]^{\mathrm{T}}$, the state space models of the plan and the desired dynamics are respectively represented as follows:

$$
\begin{aligned}
& {\left[\begin{array}{c}
\dot{x}_{1} \\
\dot{x}_{2}
\end{array}\right]=\left[\begin{array}{cc}
0 & 1 \\
0 & -n^{2} \frac{K_{t} K_{v}}{R J_{e}}
\end{array}\right]\left[\begin{array}{l}
x_{1} \\
x_{2}
\end{array}\right]-\left[\begin{array}{c}
0 \\
\frac{1}{J_{e}}
\end{array}\right] W \sin x_{1}+\left[\begin{array}{c}
0 \\
\frac{1}{J_{e}}
\end{array}\right] T_{h}+\left[\begin{array}{c}
0 \\
\frac{n K_{t}}{R J_{e}}
\end{array}\right] u+\left[\begin{array}{c}
0 \\
\frac{1}{J_{e}}
\end{array}\right] T_{d}} \\
& \triangleq A x-b_{0} W \sin x_{1}+b_{0} T_{h}+b u+b_{0} T_{d} \\
& {\left[\begin{array}{l}
\dot{x}_{1} \\
\dot{x}_{2}
\end{array}\right]=\left[\begin{array}{cc}
0 & 1 \\
0 & -2 \zeta \omega_{0}
\end{array}\right]\left[\begin{array}{l}
x_{1} \\
x_{2}
\end{array}\right]-\left[\begin{array}{c}
0 \\
\omega_{0}^{2}
\end{array}\right] \sin x_{1}+\left[\begin{array}{l}
0 \\
G
\end{array}\right] T_{h}}
\end{aligned}
$$

It should be noticed that the disturbance fulfills the matching condition. That is, the desired dynamics prescribed by Eq.(10) can be achieved because of the invariance property of sliding mode. However, when the arm hits something by accident, the operator should notice the accident by feeling the reaction torque. To this end, the system state must not in the sliding mode but in the reaching phase. Thus we will consider the desired dynamics in the reaching phase. Since the reaching phase is used in an emergency and does not have the invariance property unlike the sliding mode, the desired dynamics in the reaching phase should be linear in order to use fruitful linear robust control theories. Therefore the following linear model, which is indeed a state variable expression of Eq.(6), is chosen as a desired dynamics in the reaching phase.

$$
\left[\begin{array}{l}
\dot{x}_{1} \\
\dot{x}_{2}
\end{array}\right]=\left[\begin{array}{cc}
0 & 1 \\
-\omega_{0}^{2} & -2 \zeta \omega_{0}
\end{array}\right]\left[\begin{array}{l}
x_{1} \\
x_{2}
\end{array}\right]+\left[\begin{array}{l}
0 \\
G
\end{array}\right] T_{h}
$$

\section{Controller design}

\subsection{Reaching phase of ISMC}

In this subsection, we will deal with not power assist systems but more general nonlinear systems of the form.

$$
\dot{x}=f(x)+b u+h d=f(x)+b u+\eta b d
$$

where $\{x, f, b, h\} \in \mathbb{R}^{n},\{u, d, \eta\} \in \mathbb{R}^{1}, d$ comprises the perturbation due to external disturbances and model uncertainty, $\eta$ is a constant, and the second equation implies the 
matching condition holds. Although the original ISMC proposed by Utkin et al. ${ }^{(8),(9)}$ can be applied to a larger class of nonlinear systems, it is rather difficult to analyze the dynamics in reaching phase.

A nominal control, which can achieve an ideal dynamics without the unknown perturbation $d$, is assumed to be given and denoted by $u_{0}$. Thus, we will design another component of the control input, which is a discontinuous function to generate the sliding mode and reject the perturbation during the sliding mode. To this end, the linear switching function is defined by

$$
\sigma=\varphi^{T}(x-z)
$$

where $\varphi \in \mathbb{R}^{n}$, and $z \in \mathbb{R}^{n}$ denotes an auxiliary variable defined by

$$
\dot{z}=f(x)+b u_{0}
$$

Consequently the control input consists of the nominal control and the discontinuous control which depends on the sign of switching function as follows:

$$
u=u_{0}-\gamma \operatorname{sgn}(\sigma) \triangleq u_{0}+u_{1}
$$

Then the time derivative of the switching function is

$$
\begin{aligned}
\dot{\sigma} & =\varphi^{T}(\dot{x}-\dot{z}) \\
& =\varphi^{T}\left[f(x)+b\left(u_{0}+u_{1}\right)+\eta b d-f(x)-b u_{0}\right] \\
& =\varphi^{T} b\left(u_{1}+\eta d\right)
\end{aligned}
$$

Assuming $\varphi^{T} b \neq 0$, from $\dot{\sigma}=0$, we get the equivalent control, $u_{\text {leq }}=-\eta d$. Thus the discontinuous control cancels the perturbation and the nominal control can achieve the desired dynamics during sliding mode.

On the other hand, the dynamics in the reaching phase is obtained by substituting Eq.(15) into Eq.(12). It is clear this dynamics is the same as the sliding mode except that there exist the perturbation and a constant control, $\gamma$ or $-\gamma$. As mentioned earlier, we need to design an arbitrary dynamics in the reaching phase. To this end, adding a new component, $u_{2}$, to the control input given by Eq.(15), it follows

$$
u=u_{0}+u_{1}+u_{2}
$$

where $u_{2}$ is a function of the plant state $x$. Then, substituting Eq.(17) into Eq.(12), the dynamics in the reaching phase is

$$
\dot{x}=f(x)+b\left(u_{0}+u_{2}\right)+b[-\gamma \operatorname{sgn}(\sigma)+\eta d]
$$

Depending on the desired dynamics, there are many design methods with respect to $u_{2}$. For our application, the feedback linearization technique will be employed in the next subsection.

Now we should discuss the sliding mode when using the new control law given by Eq.(17). From the time derivative of the switching function,

$$
\begin{aligned}
\dot{\sigma} & =\varphi^{T}(\dot{x}-\dot{z}) \\
& =\varphi^{T}\left[f(x)+b\left(u_{0}+u_{1}+u_{2}\right)+\eta b d-f(x)-b u_{0}\right] \\
& =\varphi^{T} b\left(u_{\text {leq }}+u_{2}+\eta d\right)=0
\end{aligned}
$$

the equivalent control can be obtained as follows:

$$
u_{\text {leq }}=-u_{2}-\eta d
$$

This implies the discontinuous control cancels not only the perturbation but also $u_{2}$, the sliding mode is the same as the case without $u_{2}$. It should be noticed that $u_{2}$ is not used in the dynamics of the auxiliary variable. For this reason, $u_{2}$ can be seen as a matched perturbation, and the equivalent control includes $u_{2}$ given in Eq.(20).

Assuming $\varphi^{T} b>0$, the condition for the sliding mode to exist is given by

$$
\gamma>\left|u_{\text {leq }}\right|_{\text {max }}=\left|u_{2}+\eta d\right|_{\text {max }}
$$

in a sense of Lyapunov's stability, when the function $V=\sigma^{2} / 2$ is used as a candidate of the Lyapunov's functions. From this condition, $u_{2}$ should be designed not to be large in 
order to avoid larger relay gain yielding chattering.

In general siding mode control, it is common technique to improve the dynamics of switching function in the reaching phase such as a proportional reaching law, $\kappa \sigma$. In such a case, the additional component of the control input becomes a function of not only the plant state variable $x$ but the auxiliary variable $z$, since the switching function $\sigma$ includes the auxiliary variable $z$. Consequently the system dynamics in the reaching phase is more complex than the case that a function of the plant state is used as an additional component of the control. This problem is under investigation.

\subsection{Controller design for power assist system}

In this subsection, the controller proposed in the previous subsection will be applied for the power assist system under consideration.

Comparing the desired model given by Eq.(10) with the nominal plant model given by Eq.(9) where $T_{d}=0$, the nominal control is obtained as follows:

$$
u_{0}=\frac{R}{n K_{t}}\left\{\left(W-J_{e} \omega_{0}^{2}\right) \sin x_{1}+\left(n^{2} \frac{K_{t} K_{v}}{R}-2 J_{e} \zeta \omega_{0}\right) x_{2}+\left(J_{e} G-1\right) T_{h}\right\}
$$

With this nominal control and the nominal plant model, the auxiliary variable is defined by

The discontinuous control is given by

$$
\dot{z}=A x-b_{0} W \sin x_{1}+b_{0} T_{h}+b u_{0}
$$

$$
u_{1}=-\gamma \operatorname{sgn}(\sigma)
$$

where the switching function, $\sigma$ is defined by Eq.(13).

Although the desired dynamics in the reaching phase has already been determined by Eq.(11), the actual dynamics also includes the disturbance and the discontinuous control, resulting in

$$
\left[\begin{array}{l}
\dot{x}_{1} \\
\dot{x}_{2}
\end{array}\right]=\left[\begin{array}{cc}
0 & 1 \\
-\omega_{0}^{2} & -2 \zeta \omega_{0}
\end{array}\right]\left[\begin{array}{l}
x_{1} \\
x_{2}
\end{array}\right]+\left[\begin{array}{l}
0 \\
G
\end{array}\right] T_{h}+b_{0} T_{d}+b u_{1}
$$

Substituting Eq.(17) into Eq.(9) with Eq.(22), and equating the resultant equation to Eq.(25), $u_{2}$ is obtained as

$$
u_{2}=\frac{R J_{e}}{n K_{t}} \omega_{0}^{2}\left(\sin x_{1}-x_{1}\right)
$$

This is indeed a feedback linearization. Therefore $u_{2}$ is almost zero near the equilibrium point but increases as the plant state is far from the equilibrium point. Actually, since the existence condition of the sliding mode corresponding to Eq.(21) is given by

$$
\gamma>\left|u_{2}+\frac{R}{n K_{t}} T_{d}\right|_{\max }=\frac{R}{n K_{t}}\left|J_{e} \omega_{0}^{2}\left(x_{1}-\sin x_{1}\right)+T_{d}\right|_{\max }
$$

the gain $\gamma$ should be determined taking into account of the operation range with respect to the arm angle $x_{1}$.

\section{Numerical simulation}

In order to demonstrate the performance of the proposed controller, numerical simulations were carried out. It is assumed that all the state variables and the operation torque are accessible for implementation. If it is not so, state and disturbance observers can be employed ${ }^{(10)}$. The parameter values used in the simulations are the same as those of an experimental setup which is in the make in our laboratory.

$J_{e}=0.456 \mathrm{Kgm}^{2}, n=162, \quad R=1.6 \Omega, \quad W=8.61 \mathrm{Nm}, \quad K_{t}=2.5 \times 10^{-2} \mathrm{Nm} / \mathrm{A}$, $K_{v}=2.5 \times 10^{-2} \mathrm{Vs} / \mathrm{rad}, \quad m=2 \mathrm{Kg}, l=0.4 \mathrm{~m}, \quad\left|T_{d}\right|_{\max }=0.366 \mathrm{Nm}$,

where the disturbance is assumed to be Coulomb friction force in the reduction gears.

Figure 2 shows the gain curves of the ideal dynamics given by Eq.(5) and the practical desired dynamics given by Eq.(6), where $\alpha=1$ means no assist, $\alpha=0.5$ means the power amplification factor is $1 / \alpha=2$. Therefore the increase of gain shows the effect of power assist. The practical desired property shown by the solid line was determined so as to 


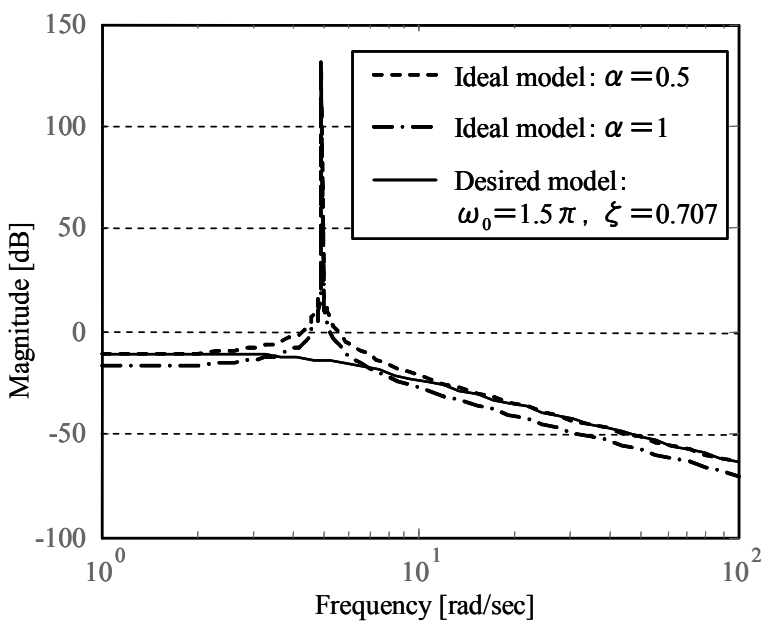

Fig.2 Gain curves of linear models

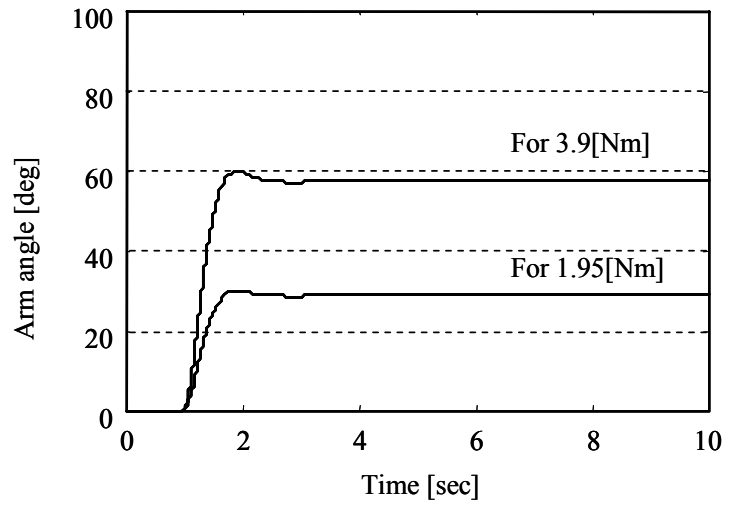

(a) Arm angles

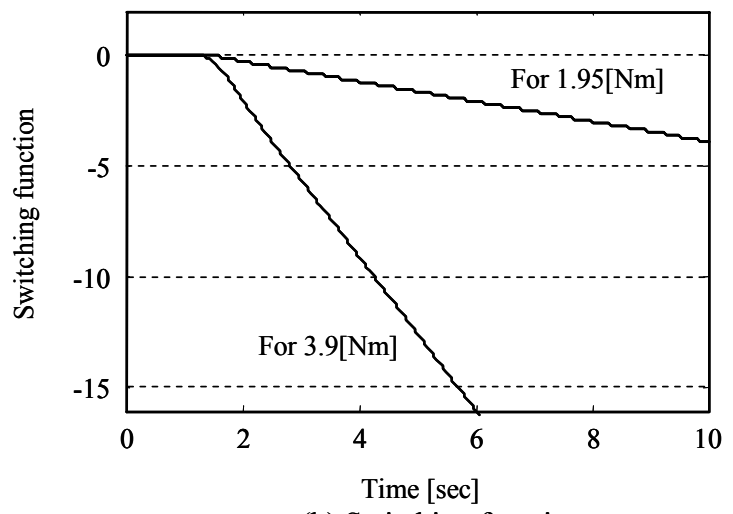

(b) Switching functions

Fig.3 Step responses during reaching phase

approximate the ideal dynamics with $\alpha=0.5$ by the stable model given by Eq.(6), where $G=5.665, \omega_{0}=1.5 \pi, \zeta=0.707$. The relay gain and the switching function were designed to guarantee the existence of sliding mode for $\left|x_{1}\right| \leq \pi / 2$, resulting in $\gamma=3$, $\varphi^{T}=\left[\begin{array}{ll}0 & 1\end{array}\right]$.

Figure 3 shows the step responses in the reaching phase, where there is no disturbance and discontinuous control to enforce the system state in the reaching phase. It can be seen from this figure that the dynamics has a homogeneity which is one of linear properties, since the step response for the operation torque $3.9 \mathrm{Nm}$ is just two times of that for the operation torque 1.95Nm. As shown in Fig.4, on the other hand, the sliding mode does not have a homogeneity, which implies the gravitational effect is realized in the sliding motion 
as desired.

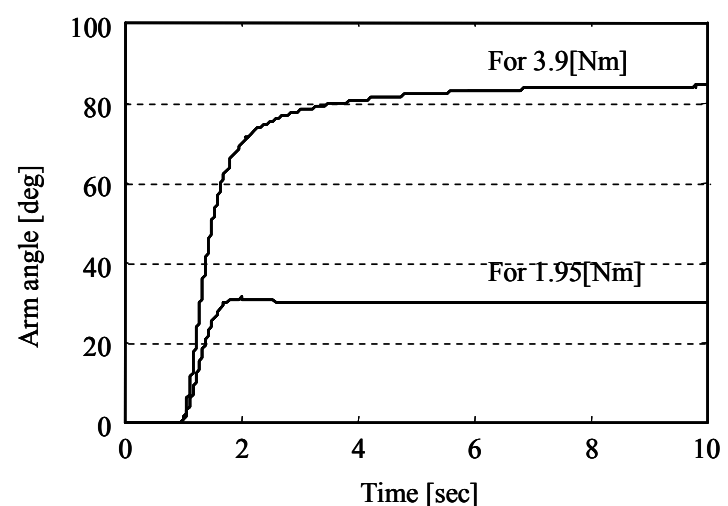

(a) Arm angles

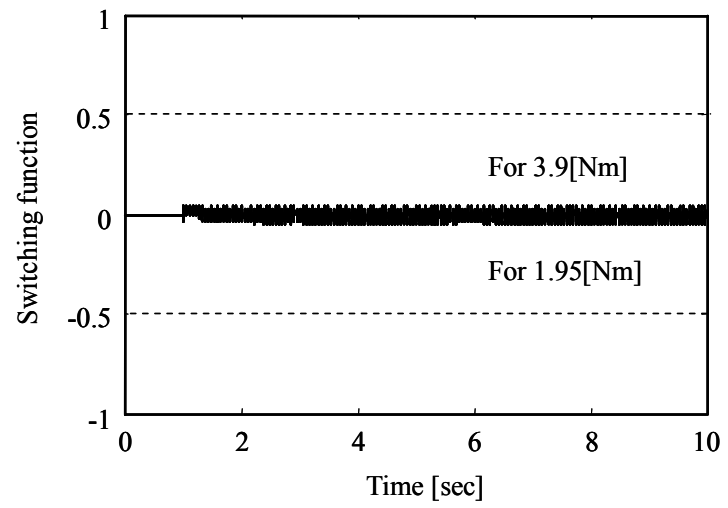

(b) Switching functions

Fig.4 Step responses during sliding mode

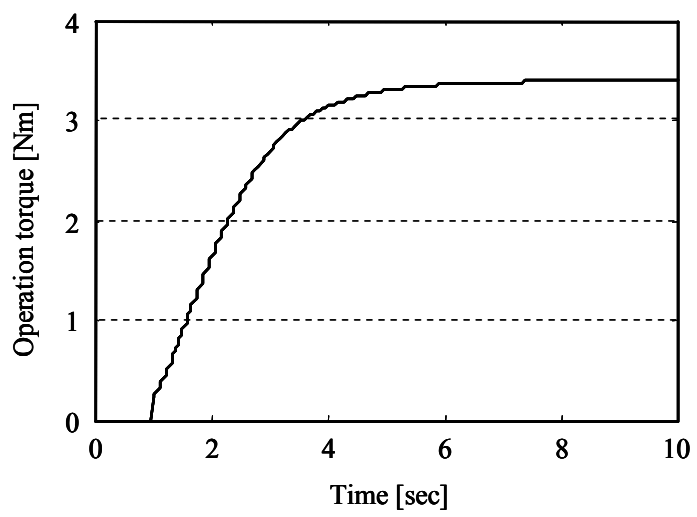

Fig.5 Operation torque

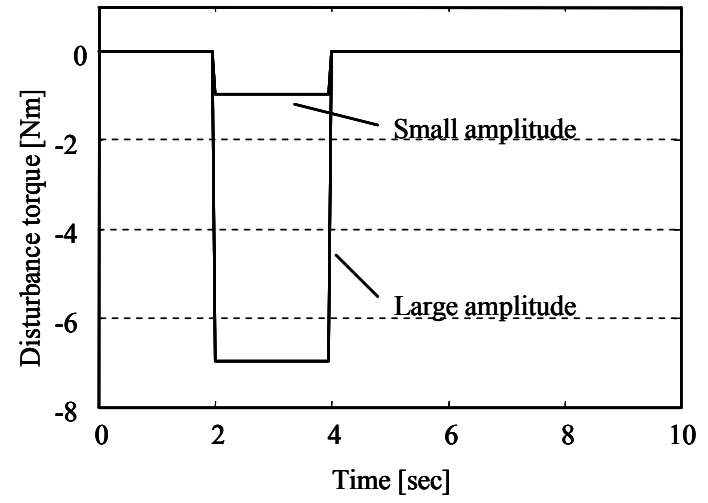

Fig.6 Disturbance torque 


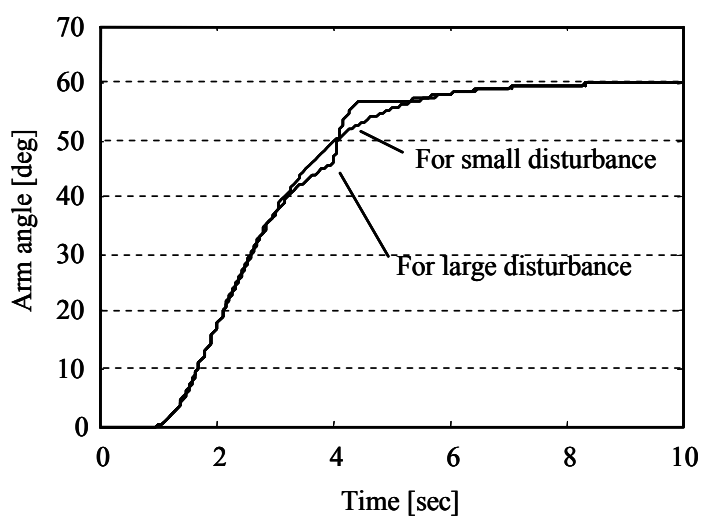

Fig.7 Arm angles

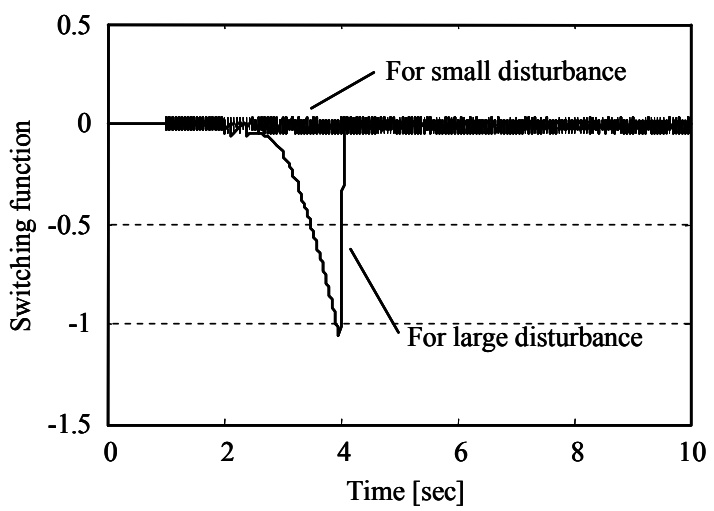

Fig.8 Switching functions

Next, the disturbance transfer characteristics depending on the amplitude of the disturbance will be investigated. The operation torque and the disturbances are shown in Figs. 5 and 6 respectively. Fig.7 shows the responses of the arm angle. As shown in Fig.8, the switching function for the larger disturbance is not confined to zero. This implies that the state trajectory is not enforced to the switching surface due to the disturbance. That is, the system is not sliding mode but in the reaching phase for a while, resulting in the fluctuations of the arm angle. In practice, This implies that the operator can feel this large disturbance.

\section{Conclusion}

In this paper, we presented a sliding mode controller utilizing the reaching phase for the power assist systems. The system is robust against the disturbance with small amplitude such as friction force since the sliding mode is maintained. On the other hand, when the arm hits something by accident, yielding a disturbance with large amplitude, the system state is not sliding mode but in the reaching phase where the system has a stable linear dynamics. Thus the operator can notice the accident by feeling the reaction torque. Although the plant was assumed to be a simple manipulator with a single degree of freedom, the proposed control law can be generalized to other systems of multi degree of freedom. Furthermore the basic idea of using the reaching phase positively may be used for quite different systems from the power assist systems, since it is natural to make the advantage of 'variable structure' systems having several dynamics inherently. Other applications are under investigation.

\section{References}

(1) Kazerooni, K., Human-robot interaction via the transfer of power and information signals, IEEE Trans. On Systems, Man and Cybernetics, Vol. 20, No. 2 (1990), pp. 450-463 
(2) Sorenson, E. A., et al., Experimental testing of a power-assisted space suit glove joint, Proc. of IEEE International Conf. on Systems, Man and Cybernetics(1997), pp.2619-2625

(3) Lee, H. et al., Control of mobile manipulators for power assist systems, Journal of Robotic Systems, Vol. 17, No. 9 (2000), pp. 469-477

(4) Fujii, F. and Wada, K., Control System Design for the Easy to Manipulate Electrically Powered Wheelchair, Trans. of the Japan Society of Mechanical Engineers, Series C, Vol.66, No.645 (2000), pp. 229-235

(5) Aoshima, S. et al., Proposal and Simulation of One-Hand Wheelchair with Power Assist Function, Trans. of the Japan Society of Mechanical Engineers, Series C, Vol.63, No.611 (1997), pp. 74-81

(6) Hayashibara, Y. et al., Study on Power Assist Systems, Trans. of the Japan Society of Mechanical Engineers, Series C, Vol.61, No.591 (1995), pp. 196-203

(7) Ikeura, R., Modeling for Cooperative Systems of Human and Robot, Journal of the Robotics Society of Japan, Vol.18, No.3(2000), pp.23-28

(8) Utkin, V. and Shi, J., Integral sliding mode in systems operating under uncertain conditions, Proc. of the 35th CDC(1996-11), pp. 4591-4596

(9) Utkin, V. et al., Sliding Mode Control in Electromechanical Systems, (1999), pp.115-129, Taylar \& Francis Ltd.

(10) Endo, G. and Yokoyama, M., Sliding Mode Control of Power-assist Systems, Proceedings of the 47th Joint Conference of Automatic Control, No.04-05 (2004-11), No.13-235 